\title{
Successful treatment of nonuniting fracture ICNF with Teriparatide in young adult osteopenic male
}

\author{
Sujoy Kundu* \\ Consultant Orthopedics, Residency Chamber, Mahendra Bhatcharya Road, Santragachi, Howrah, West Bengal, India \\ Received: 06 December 2017 \\ Accepted: 16 January 2018 \\ *Correspondence: \\ Dr. Sujoy Kundu, \\ E-mail: sujoy_doctor@rediffmail.com \\ Copyright: ( $)$ the author(s), publisher and licensee Medip Academy. This is an open-access article distributed under \\ the terms of the Creative Commons Attribution Non-Commercial License, which permits unrestricted non-commercial \\ use, distribution, and reproduction in any medium, provided the original work is properly cited.
}

\begin{abstract}
We present a case of fracture intra capsular neck of femur in a young adult osteopenic patient, who had a nonunion and early signs of avascular necrosis even after the initial surgical intervention. Teriparatide was administered for two monthly doses after diagnosis of nonunion and avascular necrosis of femoral neck. 90\% successful union was achieved in 2 months without further surgical intervention, and no adverse events related to the use of teriparatide were observed. Our case report shows that teriparatide can be an alternative to surgical intervention in nonunion of ICNF with early signs of avascular necrosis.
\end{abstract}

Keywords: Teriparatide, Nonunion, Avascular necrosis, Intracapsular fracture neck femur, Accelerated fracture healing, Osteopenic

\section{INTRODUCTION}

Femoral neck fractures in young adults are uncommon and often occur due to high-energy trauma. ${ }^{1}$ They are commonly associated with femoral head osteonecrosis and nonunion. The reported risk of avascular necrosis (AVN) following intracapsular neck of femur (ICNF) fractures is about 1 in 5 in those younger than 60 years of age, $12.5 \%$ in those aged $60-80$ years and $2.5 \%$ for those aged more than 80 years. $^{2}$ The key factors in treating femoral neck fractures include early diagnosis, early surgery, anatomic reduction, capsular decompression and stable internal fixation.

Physiological fracture healing involves rapid bone regeneration and osteoclast-mediated remodeling resulting in new bone thus resuming structural integrity and a geometrical configuration like before. Nonunion represents poor healing since complete restoration fails. It indicates that the normal biologic healing process is deficient in achieving complete repair. Nonunion and avascular necrosis of the femoral head or a combination of both is the main complication following fractures of the femoral neck. In spite of improved operative techniques, nonunion is still reported in $10-20 \%$ of cases. $^{3}$

The economic impact of nonunion is significant especially for an earning member of family in terms of loss of work days. Nowadays in cases of nonunions of the femoral neck, the common approach is prosthetic replacement of the hip, more so if there is also evidence of avascular necrosis of the head of femur. This leads to rapid pain relief and early mobilization. But long-term results of hip arthroplasties, especially in younger people and in the presence of osteopenia, are not satisfactory; and a less radical approach is desirable. Any medical therapy which promotes healing in nonunion cases or shortens the time interval for fracture healing will be of great social and economic benefit.

Teriparatide (rhPTH[1-34]) is a peptide representing the amino-terminal portion of human parathyroid hormone. It 
is a skeletal anabolic agent approved for the treatment of osteoporosis. In terms of fracture healing, the accelerating effect of teriparatide has been reported in animal model and in some clinical studies. Femoral nonunion is challenging for the patient as well as the surgeon. To date, it has been treated by various surgical interventions, with only minimal focus on medical approaches.

We medicated teriparatide in a young adult osteopenic patient, who had a nonunion of the femur even after the initial surgical intervention after 3 months. Teriparatide was administered for two doses (monthly interval) after a diagnosis of nonunion and avascular necrosis of femoral neck. A successful union was obtained after 2 months without further surgical intervention, and there were no adverse events related to the use of teriparatide. Our report showed that teriparatide could be an alternative to surgical intervention in nonunion of the intra capsular neck of femur.

\section{CASE REPORT}

A 39-year-old gentleman sustained a closed fracture presented to our tertiary care center after a mechanical fall on right hip. $\mathrm{He}$ is an active, independent gentleman with no significant co-morbidities. His pre-morbid mobility was good and he did not require any walking aids.

He was immediately resuscitated. On clinical examination he had a tender right hip with a shortened and externally rotated leg. A plain antero-posterior radiograph and oblique view of the hip confirmed the presence of garden grade 4 fracture. Clinical examination revealed external rotation of right leg and pain on passive movement of right hip. X-ray of his pelvis showed completely displaced intracapsular hip fractures on right side. (Garden grade IV fracture).

After careful pre-operative planning, fracture was reduced by LEADBETTER (In flexion) method. After accurate reduction, rigid internal fixation under $\mathrm{x}$ ray control was done using three compression hip screws (CHS) to prevent rotation of the proximal fragment. The threaded portions of the screws were seen to cross the fracture line to get a better lag effect. This was undertaken with the patient in the supine position within 4 days of accident using an antero-lateral approach by a senior orthopaedic surgeon (SK). X-ray showed a satisfactory position of the fracture post surgery. He had a satisfactory post-operative recovery and was able to mobilize and fully weight-bear within 2 days of the surgery.

The patient was kept in the bed supine with a pillow under the knee, antibiotics \& analgesics were given for a week post operatively. Patient was mobilized in the bed with quadriceps exercises. Sutures were removed on the 10th day. Non-weight crutch walking was advised after reduction of pain in the operated area. Monthly check-ups were planned clinically and radiological until the union of fracture. Full weight bearing was to be allowed only after definite radiological evidence of union. Harris hip score was used to assess functional outcome.

After 3 months the fracture seemed securely fixed on radiographs. However, there were findings of nonunion i.e. persistent gap between fragments, sclerosis of fracture margin, absence of callus formation and absence of bone bridging the fracture site. A diagnosis of nonunion was made. There was no clinical sign of infection at the fracture site, like swelling, local heat, or redness. Laboratory tests, including WBC, C-reactive protein, and ESR, were normal. Thus, infected nonunion was ruled out. The patient was non-smoker and non-alcoholic, and had no history of metabolic disease or glucocorticoid use.

Other laboratory tests indicative of defective bone healing, including serum alkaline phosphatase, PTH, calcium, phosphorus, and $25-\mathrm{OH}$ vitamin D were normal. The patient denied to undergo another operation. But agreed for off-label teriparatide therapy at doses approved for the treatment of osteoporosis. Two doses of monthly teriparatide were administered Moreover the intention of the treating surgeon was to avoid high cost of 2 nd operation, morbidity, chances of infection, avoiding bed ridden condition and possible AVN. At $5^{\text {th }}$ month $90 \%$ union was observed on $\mathrm{X}$-ray and patient improved symptomatically.

\section{DISCUSSION}

Nonunion of a femoral fracture is defined as a persistent fracture after 3 months without evidence of healing. Risk factors identified for delayed or impaired union of fractures include advanced age, diabetes, corticosteroid treatment, and osteoporosis. In addition, mechanical and anatomical factors such as instability and poor bone-tobone contact are other contributing factors. Abnormal levels of calcium, vitamin $\mathrm{D}$, and parathyroid hormone (PTH) also impact fracture healing because of their role in bone metabolism.

Bone is a dynamic tissue at the microscopic level despite appearing to be a static organ macroscopically. Parathyroid hormone has an important role in bone metabolism and helps maintain calcium homeostasis in both intra and extracellular fluids. Intermittent exposure to PTH elicits an anabolic response while continuous exposure results in a catabolic cascade. This important discovery eventually led to the therapeutic development of teriparatide [rhPTH (1-34)] taking advantage of the anabolic effect of PTH on bone. It is the only currently available anabolic agent to stimulate osteoblast activity. ${ }^{4}$ Teriparatide has been shown to increase bone mass, improve bone quality, and reduce the risk of fracture in patients with severe osteoporosis. ${ }^{5}$ Several reports have demonstrated the efficacy of the daily administration of recombinant TPTD in the treatment of nonunion 
fractures; The anabolic effect of the once in a month dose of TPTD is thus presented in our case study. ${ }^{6}$

An important objective in the treatment of an intracapsular fracture of the hip is to obtain stable osseous support of the femoral head on the femoral neck. Thus screw fixation is used to increase stability by compressing the fracture and then maintaining the reduction by neutralizing forces acting on the hip. The absence of callus formation as seen on 3 month X-ray in our case, it can be termed as a nonunion. In our study, evidence of nonunion were present at 3 months after which teriparatide was initiated and after 2 months of its administration $90 \%$ of the fracture united as seen on radiograph. Clinically also, the patient showed improvement. Few other reports in the past have suggested that teriparatide may improve fracture healing in humans. Coppolo et al described 4 cases of open fracture nonunion of lower limb, all improved clinical and radiographic outcomes of the fracture, and the patient regained normal routine within 3 months. ${ }^{7}$ Chintamaneni et al described the first case of sternal fracture nonunion responding to treatment with teriparatide, with dramatic radiographic healing more than 6 months after the initial fracture. ${ }^{8}$ Another study showed a patient with delayed union of a humeral shaft fracture healed after 5 months of therapy with teriparatide, without other interventions. ${ }^{9}$ Resmini et al described a 79-year-old osteoporotic lady already on teriparatide treatment who sustained a fracture of the left proximal humerus. ${ }^{10}$ She was maintained on teriparatide, and radiographic fracture healing occurred rapidly after 25 days which was attributed to teriparatide treatment. Overall, these patients were suspected to experience accelerated healing with teriparatide and an earlier return to normal functioning. Overall, these results suggest that teriparatide may have a role in accelerating fracture healing.

To the best of our knowledge, our case is the first case reported in literature of a fracture nonunion ICNF and initial phase of avascular necrosis responding to treatment with teriparatide. Our case differs from prior reports which involved use of teriparatide during the acute phase of fracture healing. Using teriparatide in acute phase may have confounding variable that these fractures may have otherwise healed even without teriparatide. Our patient's dramatic radiographic healing occurred more than 3 months after the initial fracture. It is highly unlikely that our patient's fracture would have healed spontaneously with continued observation, not requiring a second surgery. Thus our case puts forth a convincing evidence that teriparatide may be a useful treatment for nonunion of fractures, also in presence of avascular necrosis. Further well-designed studies are needed to evaluate the efficacy of teriparatide in fracture healing and in the setting of nonunions.

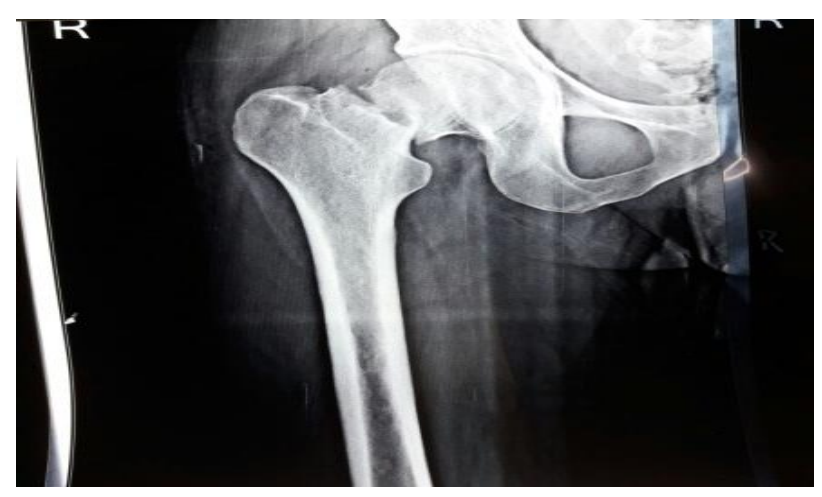

Figure 1: Garden grade 4 fracture: pre-operative Xray.

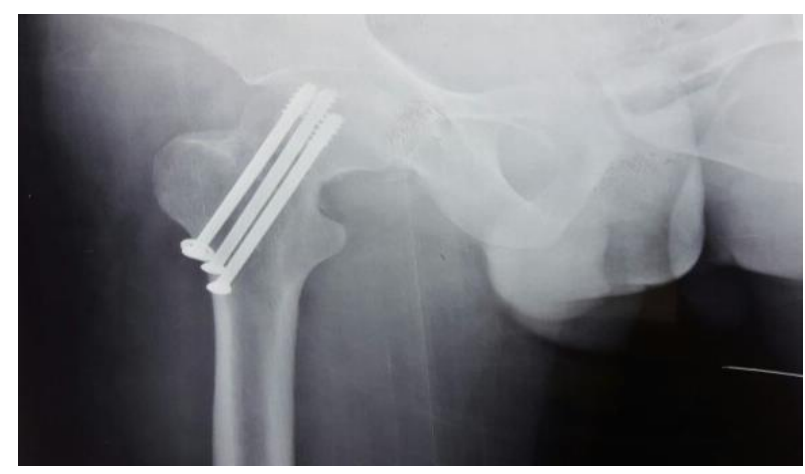

Figure 2: Immediate post-operative X-ray (fracture reduced and internally fixed with $3 \mathrm{CHS}$ ).

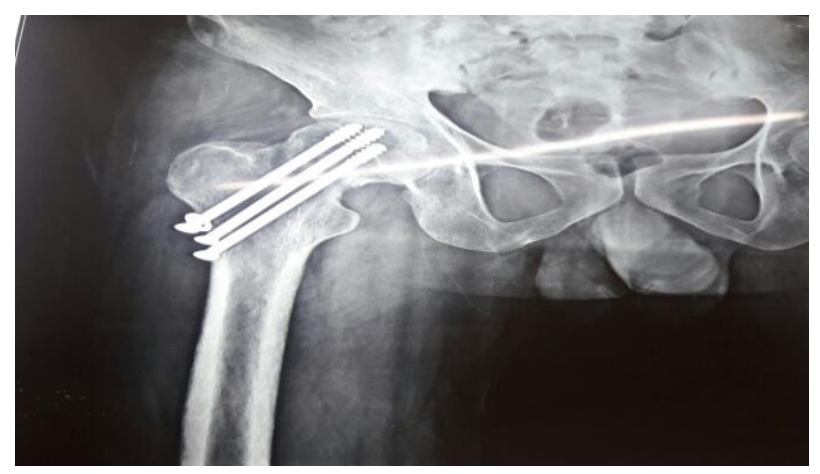

Figure 3: 3 month X-ray showing no evidence of callus formation.

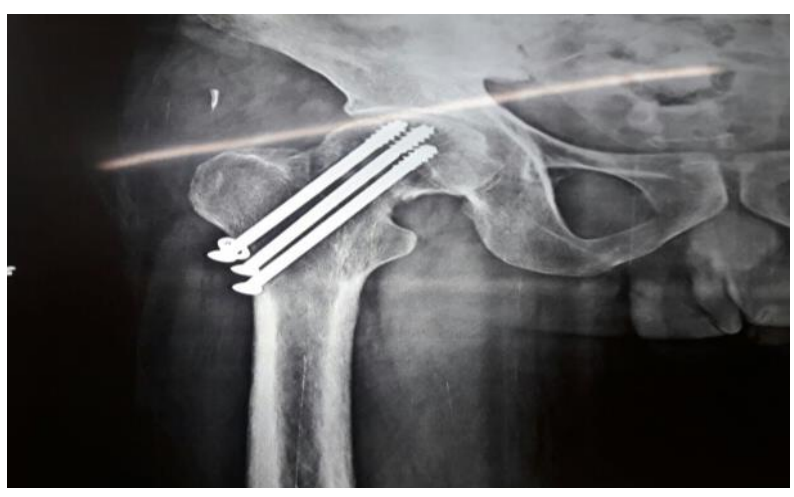

Figure 4: 4 month X-ray showing mild signs of AVN. 

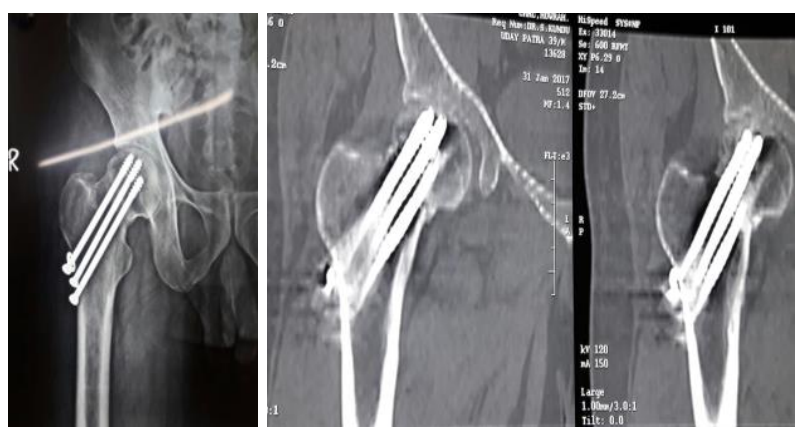

Figure 5: 5 month X-ray and MRI showing 90\% union.

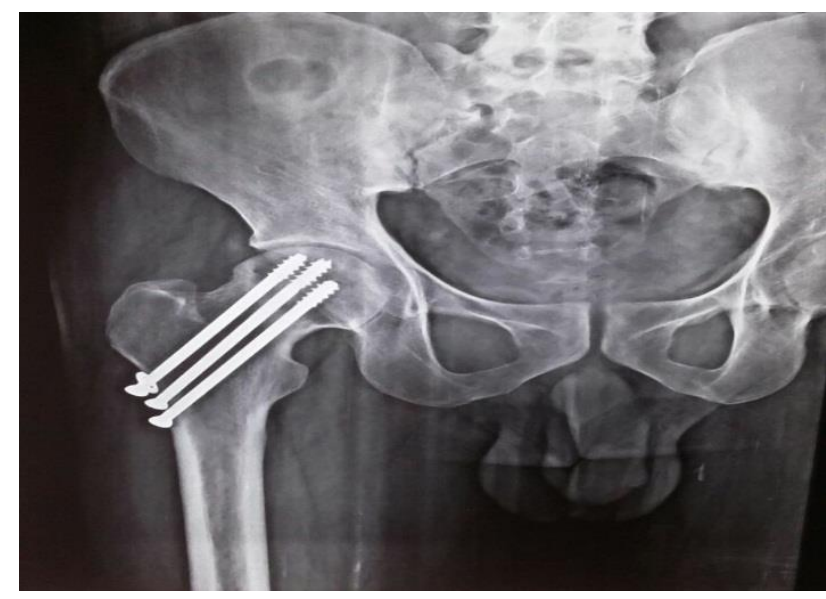

Figure 6: More than a year after fracture X-ray showing complete union.

Funding: No funding sources Conflict of interest: None declared

Ethical approval: Not required

\section{REFERENCES}

1. Robinson CM, Court-Brown CM, McQueen MM, Christie J. Hip fractures in adults younger than 50 years of age. Epidemiology and results. Clin Orthop Relat Res. 1995;(312):238-46.
2. Ravikumar K.J., Marsh G. Internal fixation versus hemiarthroplasty versus total hip replacement for displaced sub capital fractures of femur. 13 year results of prospective randomised study. Injury. 2003;31:793-7.

3. Mathews V, Cabanela ME. Femoral neck nonunion treatment. Clin Orthop Relat Res. 2004;419:57-64.

4. Borggrefe J, Graeff C, Nickelsen TN, Marin F, Glüer CC. Quantitative computed tomographic assessment of the effects of 24 months of teriparatide treatment on 3D femoral neck bone distribution, geometry, and bone strength: results from the EUROFORS study. J Bone Miner Res. 2010;25(3):472-81.

5. Sibai T, Morgan EF, Einhorn TA. Anabolic agents and bone quality. Clin Orthop Relat Res. 2011;469(8):2215-24.

6. Lee YK, Ha YC, Koo KH. Teriparatide, a nonsurgical solution for femoral nonunion? A report of three cases. Osteoporos Int. 2012;23(12):2897900 .

7. Coppola C, Del Buono A, Maffulli N. Teriparatide in Fracture Non-Unions. Translational Medicine @ UniSa. 2015;12:47-53.

8. Chintamaneni S, Finzel K, Gruber BL. Successful treatment of sternal fracture nonunion with teriparatide. Osteoporos Int. 2010;21(6):1059-63.

9. Oteo-Alvaro A, Moreno E. Atrophic humeral shaft nonunion treated with teriparatide (rh PTH 1-34): a case report. J Shoulder Elbow Surg. 2010;19(7):e228.

10. Resmini G, Iolascon G. 79-year-old postmenopausal woman with humerus fracture during teriparatide treatment. Aging Clin Exp Res. 2007;19(4):30-1.

Cite this article as: Kundu S. Successful treatment of nonuniting fracture ICNF with Teriparatide in young adult osteopenic male. Int J Res Orthop 2018;4:326-9. 